\title{
A comparison of the effects of manual hyperinflation and ventilator hyperinflation on restoring end-expiratory lung volume after endotracheal suctioning: a pilot physiologic study
}

Matthew P. Linnane BPhty ${ }^{\mathrm{a}, \mathrm{b},{ }^{*}}$ matthew.linnane@health.qld.gov.au, Lawrence R. Caruana BPhty ${ }^{\mathrm{a}, \mathrm{b}}$ Lawrence.Caruana@health.qld.gov.au, Oystein Tronstad BPhty ${ }^{\mathrm{a}, \mathrm{b}}$

Oystein.Tronstad@health.qld.gov.au, Amanda Corley RN, BN, GradCertHSci ${ }^{a}$

Amanda.Corley@health.qld.gov.au, Amy J. Spooner RN, BN, GradDipICU ${ }^{c}$

Amy.Spooner@qut.edu.au, Adrian G. BarnettBSc(Hon), PhD ${ }^{c}$ a.barnett@qut.edu.au, Peter J. Thomas BPhty (Hons) PhD ${ }^{\mathrm{d}}$ PeterJ.Thomas@health.qld.gov.au, James R. Walsh, BPhty PhD

James.Walsh@health.qld.gov.au

${ }^{a}$ Critical Care Research Group, The Prince Charles Hospital and University of Queensland, Brisbane, Qld, Australia, 4032

${ }^{\mathrm{b}}$ Physiotherapy Department, The Prince Charles Hospital, Brisbane, Qld, Australia, 4032

${ }^{\mathrm{c}}$ Institute of Health and Biomedical Innovation, School of Public Health, Queensland University of Technology, Brisbane, Qld, Australia, 4000

${ }^{\mathrm{d}}$ Physiotherapy Department, Royal Brisbane and Women's Hospital, Brisbane, Qld, Australia, 4006

${ }^{\mathrm{e}}$ School of Allied Health Sciences, Griffith University, Gold Coast, Qld, Australia, 4215

"Corresponding author at: Physiotherapy Department, The Prince Charles Hospital, Rode Road,

Chermside, 4032, Queensland, Australia. 


\section{$\underline{\text { Abstract }}$}

Purpose: Endotracheal suctioning (ES) of mechanically ventilated patients decreases end-expiratory lung volume (EELV). Manual hyperinflation (MHI) and ventilator hyperinflation (VHI) may restore EELV post-ES but it remains unknown which method is most effective. The primary aim was to compare the efficacy of MHI and VHI in restoring EELV post-ES.

Materials and Methods: ES was performed on mechanically ventilated intensive care patients, followed by MHI or VHI, in a randomised crossover design. The washout period between interventions was one hour. End-expiratory lung impedance (EELI), measured by electrical impedance tomography, was recorded at baseline, during ES, during hyperinflation and 1, 5, 15 and 30 minutes post-hyperinflation.

Results: Nine participants were studied. ES decreased EELI by 1672 z (95\% CI, 1204 to 2140) from baseline. From baseline, MHI increased EELI by $1154 \mathrm{z}(95 \%$ CI, 977 to 1330) while VHI increased EELI by $769 \mathrm{z}(95 \% \mathrm{CI}, 457$ to 1080$)$. Five minutes post-VHI, EELI remained $528 \mathrm{z}$ (95\% CI, 4 to 1053) above baseline. Fifteen minutes post-MHI, EELI remained 351z (95\% CI, 111 to 592) above baseline. At subsequent time-points, EELI returned to baseline.

Conclusions: MHI and VHI effectively restore EELV above baseline post-ES and should be considered post suctioning. 


\section{$\underline{\text { Keywords }}$}

Ventilator Hyperinflation, Manual Hyperinflation, Mechanical Ventilation, Endotracheal Suction, Electrical Impedance Tomography, Lung Recruitment 


\section{Abbreviations}

$\mathrm{MV}=$ mechanical ventilation

$\mathrm{ES}=$ endotracheal suctioning

EELV $=$ end-expiratory lung volume

$\mathrm{MHI}=$ manual hyperinflation

VHI $=$ ventilator hyperinflation

EIT $=$ electrical impedance tomography

EELI $=$ end-expiratory lung impedance

$\mathrm{C}_{\text {stat }}=$ static lung compliance

$\mathrm{PaO}_{2} / \mathrm{FiO}_{2}=$ ratio of arterial oxygen partial pressure to fraction of inspired oxygen

$\mathrm{PIP}=$ peak inspiratory pressure

$\mathrm{SpO}_{2}=$ peripheral oxygen saturation 


\section{Introduction}

Mechanical ventilation (MV) is associated with multiple complications including ventilator induced lung injury $[1,2]$. Atelectrauma, or mechanical damage caused by cyclic opening and collapse of alveoli, significantly contributes to the development of lung injury $[2,3]$. To prevent this, it is imperative to maintain optimal lung volumes throughout the respiratory cycle $[4,5]$. Endotracheal suctioning (ES) is a fundamental procedure for removing airway secretions in mechanically ventilated patients, however, it results in a significant reduction of end-expiratory lung volume (EELV) which persists at thirty minutes post-ES [6, 7]. Moreover, ES has been associated with significant impairments in gas exchange $[6,8,9]$.

Manual hyperinflation (MHI) and ventilator hyperinflation (VHI) are interventions commonly performed with the objectives of improving oxygenation, facilitating secretion clearance and increasing EELV $[10,11]$. MHI involves disconnection from MV to deliver large tidal volumes via a manual resuscitation bag $[12,13]$. MV disconnection causes potential adverse outcomes including loss of EELV, de-oxygenation, shear stress of alveoli, transmission of infection, and inaccuracy of airway pressure, inspiratory flow and tidal volume [10,14-18]. VHI is a newer technique which mimics MHI through modification of the ventilator parameters, without MV disconnection $[18,19]$. Multiple studies [10, 15, 20,21], including a recent systematic review [11], have demonstrated that MHI and VHI have similar effects on secretion clearance, pulmonary compliance and oxygenation. However, there is a paucity of evidence to compare their efficacy in restoring EELV post-ES.

Electrical impedance tomography (EIT) is a non-invasive technique which is implemented at the bedside to provide real-time dynamic images and measures of regional lung ventilation $[22,23]$. When compared with electron beam computerised tomography, EIT demonstrates a highly significant correlation in measuring regional lung ventilation [24]. Accurate measurement of changes in lung volume using EIT is due to a strong linear relationship between the change in electrical impedance 
and the change in lung volume. Therefore, the change in end-expiratory lung impedance (EELI) correlates directly with the change in EELV $[25,26]$. Furthermore, it has been demonstrated that EIT reliably and precisely measures the change in lung volumes during ES and recruitment manoeuvres [27-29].

Following on from our previous study investigating lung volume loss during ES [7], the investigators elected to investigate methods to reverse ES-induced EELV loss. Given the lack of evidence defining the effects of MHI or VHI on increasing lung volume post-ES, the primary aim of this study was to determine which technique was better in restoring EELV post-ES. The secondary aims were to assess the differences in oxygenation, static lung compliance $\left(\mathrm{C}_{\text {stat }}\right)$ and haemodynamics following $\mathrm{MHI}$ and VHI. We hypothesised that VHI would be superior to MHI in restoring EELV, maintaining oxygenation and improving $\mathrm{C}_{\text {stat }}$ post-ES whilst having no adverse effect on haemodynamics.

\section{Material and Methods}

A prospective, randomised crossover design was conducted in the intensive care unit of a cardiothoracic tertiary hospital. The study protocol was approved by The Prince Charles Hospital Human Research and Ethics Committee (HREC/12/QPCH/284). Informed written consent was obtained from the participants or their next of kin prior to recruitment.

\section{Population}

The inclusion, exclusion and withdrawal criteria are in Table 1. Initially, the inclusion criteria were very specific to include only patients who had been ventilated on synchronised intermittent mandatory ventilation - volume control mode for 48 to 96 hours, had a ratio of arterial oxygen partial pressure to fraction of inspired oxygen $\left(\mathrm{PaO}_{2} / \mathrm{FiO}_{2}\right)$ of 200 to 350 , and were sedated to a Riker score of 2 to 3 . 
However, during the study, the restrictions on $\mathrm{MV}$ time, $\mathrm{PaO}_{2} / \mathrm{FiO}_{2}$ range and Riker score range were removed to facilitate participant recruitment. A patient flowchart through the study is in Figure 1.

\section{Protocol}

After participants were recruited to the study, MHI and VHI order was randomly allocated using sequentially numbered, opaque, sealed envelopes which were blinded to the investigators until opening. Calibration of the EIT device was performed prior to performing each study intervention. Participants were positioned supine with the bed head elevated to 30 degrees. The EIT belt was positioned at the fifth intercostal space. A twenty-minute stabilisation period was applied prior to commencement of data collection. Endotracheal suction was performed with a closed suction system (Kimvent Turbo-Cleaning Closed Suction System, Kimberly-Clark, Roswell, GA) and the size of the suction catheter was standardised (for an endotracheal or tracheostomy tube with an internal diameter of $7.0-8.5 \mathrm{~mm}$, a $12 \mathrm{~F}$ catheter was used; for an internal diameter of $9.0-9.5 \mathrm{~mm}$, a $14 \mathrm{~F}$ catheter was used). After one minute of pre-oxygenation with $100 \% \mathrm{FiO}_{2}$, suction was performed twice. Each pass of the suction catheter was of six seconds duration, with a five second interval between suctions. A continuous suction technique was used, with negative pressure of $150 \mathrm{mmHg}$ applied only during withdrawal of the catheter. MHI or VHI was then performed according to the randomisation order.

MHI was performed with a Mapleson-C circuit (Intersurgical Ltd, Workingham, Berkshire), with a manometer in line and an oxygen flow of $15 \mathrm{~L} /$ minute. To reflect clinical practice in the study intensive care unit, a positive end-expiratory pressure valve was not used. The participants received four one-minute sets consisting of eight hyperinflation breaths ( 4 sets $\times 8$ breaths $\mathrm{x} 1$ minute per set). Each hyperinflation breath consisted of a three second inspiration to a peak inspiratory pressure (PIP) of $35-40 \mathrm{~cm} \mathrm{H}_{2} \mathrm{O}$, followed by a two second inspiratory pause and passive expiration. Between each MHI set, participants received one minute of tidal breathing which matched the respiratory rate and PIP delivered by the ventilator at baseline. VHI was performed in synchronised intermittent 
mandatory ventilation - volume control mode on a Puritan Bennett 840 ventilator (Covidien, Mansfield, Mass) by increasing the $\mathrm{FiO}_{2}$ to $100 \%$, reducing the respiratory rate to eight breaths/minute and decreasing the inspiratory flow rate to $20 \mathrm{~L} /$ minute. The tidal volume was then increased until a PIP of $35-40 \mathrm{cmH}_{2} \mathrm{O}$ was reached. Once the target pressure was achieved, the tidal volume was maintained for eight breaths with an inspiratory pause of two seconds. This was repeated for a total of four one-minute sets consisting of eight hyperinflation breaths $(4$ sets $\times 8$ breaths $\mathrm{x} 1$ minute per set). Between each VHI set, participants received one minute of tidal breathing which matched the baseline ventilation parameters. Positive end-expiratory pressure and pressure support settings remained unchanged throughout the study. There was a one-hour washout period between the two hyperinflation interventions and ES was performed prior to each intervention. All ES, MHI and VHI interventions were performed by the same experienced investigator (M.L.).

\section{$\underline{\text { Measurements }}$}

Data were collected on demographic information, primary diagnosis, duration of mechanical ventilation, Acute Physiology and Chronic Health Evaluation II score and Sequential Organ Failure Assessment score. Changes in EELI/EELV were measured using EIT (Dräger PulmoVista 500, Lübeck, Germany) at baseline, during suction, during hyperinflation and at 1, 5, 15 and 30 minutes post-hyperinflation. Haemodynamic measures of heart rate, invasive blood pressure and mean arterial pressure were also recorded at these time points (Marquette monitor, GE Medical Systems Information Technologies Inc, Milwaukee, Wis). Peripheral oxygen saturation $\left(\mathrm{SpO}_{2}\right)$ via finger pulse oximetry (Marquette monitor, GE Medical Systems Information Technologies Inc, Milwaukee, Wis) and $\mathrm{C}_{\text {stat }}$ via the ventilator were measured at baseline and at 1, 5, 15 and 30 minutes posthyperinflation. Arterial blood gases were measured (ABL 800 gas analyser, Radiometer, Copenhagen, Denmark) at baseline and at 5 and 30 minutes post-hyperinflation to assess $\mathrm{PaO}_{2} / \mathrm{FiO}_{2}$ ratio. Baseline measurements of all variables were recorded prior to each delivered study intervention. 


\section{$\underline{\text { Statistical Analysis }}$}

Data were analysed using a regression model with a random intercept for each participant to control for repeated data. We used predictors of order (1 or 2), treatment group (MHI or VHI), time as a categorical variable (baseline, ES, hyperinflation, $1 \mathrm{~min}, 5 \mathrm{mins}, 15 \mathrm{mins}$ and $30 \mathrm{mins}$ ) and the interaction between treatment and time. The interaction demonstrates whether there is a difference between the two treatment groups over time. To visualise the difference, we used plots of the mean changes from baseline by treatment group over time. We did not adjust for multiple comparisons as we followed the recommendation of "simply describing what tests of significance have been performed, and why, is generally the best way of dealing with multiple comparisons" [30].

Results are expressed as mean and 95\% confidence intervals. We used $95 \%$ confidence intervals without p-values, but readers interested in statistical significance at the standard 0.05 threshold can infer significance from a confidence interval that does not contain zero.

\section{Results}

Ten participants were recruited to the study. One participant was withdrawn during data collection due to an unstable arrhythmia, as per the withdrawal criteria. Therefore, nine participants were studied. Demographic details of the participants are in Table 2 and baseline ventilator settings are in Table 3. Participants were receiving various combinations of sedative medications including fentanyl (4), midazolam (4), Propofol (2) and temazepam (2) but none were receiving neuromuscular blockers.

\section{Effect of ES on EELI}

The mean baseline EELI of all participants prior to ES was 597 no units (z) (95\% CI, -202 to 1397). ES resulted in a reduction of EELI by $1672 \mathrm{z}$ (95\% CI, 1204 to 2140), indicating a loss of lung volume. The estimated change in mean EELI from baseline during ES is shown in Figure 2. 


\section{Effect of MHI and VHI on restoring EELI post-ES}

When compared with baseline, MHI resulted in an increased EELI during hyperinflation and at 1, 5 and 15 minutes post-MHI. At 30 minutes post-MHI, EELI was close to baseline. The change in EELI from baseline post-MHI is in Table 4.

When compared with baseline, VHI resulted in an increased. EELI during hyperinflation and at 1 and 5 minutes post-VHI. At 15 and 30 minutes post-VHI, EELI was close to baseline. The change in EELI from baseline post-VHI is in Table 4.

There were two differences between the treatment groups. During hyperinflation and at 5 minutes afterwards, the MHI group had a higher EELI than the VHI group. However, between these time points (at 1 minute post hyperinflation) EELI increased post-VHI and decreased post-MHI. The change in mean EELI from baseline during hyperinflation and at each time point post MHI and VHI is in Figure 2.

There was a strong impact of hyperinflation order, with the second intervention resulting in $563 \mathrm{z}$ ( $95 \%$ CI, 472 to 655 ) less than the first intervention. As the order of treatments was randomised this difference should not impact on the estimated treatment effect.

\section{Effect of MHI and VHI on static lung compliance}

The mean baseline $\mathrm{C}_{\text {stat }}$ was $52.7 \mathrm{ml} / \mathrm{cmH}_{2} 0$ (95\% CI, 36.9 to 68.4 ). Both MHI and VHI were associated with small increases in $\mathrm{C}_{\text {stat }}$ over time when compared with baseline. At 30 minutes postMHI, it was $2.9 \mathrm{ml} / \mathrm{cmH}_{2} 0(95 \% \mathrm{CI},-3.3$ to 9.2$)$ greater than baseline while VHI resulted in an 
increase of $4.9 \mathrm{ml} / \mathrm{cmH}_{2} 0(95 \% \mathrm{CI},-8.3$ to 18.2$)$. There were no clinically important differences between interventions at any of the time points and no impact of order was demonstrated.

\section{Effect of MHI and VHI on oxygenation}

The effect of MHI and VHI on oxygenation is summarised in Table 5. At 5 minutes post-MHI there was an increase in $\mathrm{PaO}_{2}$ compared to baseline. However, 30 minutes post-MHI, $\mathrm{PaO}_{2}$ had returned to baseline. VHI resulted in very small increases in $\mathrm{PaO}_{2}$ over time and there were no differences between groups.

MHI and VHI both resulted in minor improvements in $\mathrm{PaO}_{2} / \mathrm{FiO}_{2}$ at 5 minutes post-hyperinflation when compared to baseline. After 30 minutes, MHI decreased slightly below baseline whereas VHI remained slightly above baseline.

There were no major differences in $\mathrm{SpO}_{2}$ between $\mathrm{MHI}$ and VHI at any time points. When compared to baseline, MHI resulted in a small improvement of $\mathrm{SpO}_{2}$ after 1 minute; however, this was not a clinically important change. No impact of order was demonstrated for any of the oxygenation variables.

\section{Effect of MHI and VHI on haemodynamics}

There was no clinically relevant change in heart rate from baseline at any time points or between groups. At 1 minute post-MHI, there was an increase in mean arterial pressure by $5.3 \mathrm{mmHg}(95 \%$ CI, 0.4 to 10.1), however, this was not clinically important compared to a baseline mean of 77.3 $\mathrm{mmHg}(95 \% \mathrm{CI}, 71.1$ to 83.6$)$. There was no major difference in mean arterial pressure between 
groups and VHI did not result in any important change from baseline. No impact of order was demonstrated for either of the haemodynamic variables.

\section{Discussion}

The results of this study demonstrate that ES was associated with a reduction of EELV during synchronised intermittent mandatory ventilation - volume control mode in our patient population.

Both MHI and VHI were equally effective in restoring EELV post-ES. MHI and VHI result in similar outcomes with respect to $\mathrm{C}_{\text {stat, }}$ oxygenation and haemodynamic parameters.

These results are particularly important when compared to previous studies $[6,7]$ which have demonstrated significant and sustained loss of EELV post-ES with open and closed suction systems. Heinze et al [6] and Corley et al [7] showed a reduction in EELV at twenty minutes and thirty minutes post-ES respectively. The reduction of EELV after closed ES demonstrated in this study is supportive of these findings. In contrast, other studies have shown minimal loss of lung volume post-ES [14, 29 , 31]. The study populations, suction techniques and ventilator settings were different from this study and these variations may account for conflicting outcomes. Heterogeneity in the existing evidence regarding EELV post-ES makes generalisation difficult and the interaction between patient, ventilator and suction technique must be considered. Nonetheless, it cannot be assumed that closed ES prevents suction-induced lung volume loss in all cases [7] and appropriate techniques may be implemented post-ES to restore EELV.

The most important clinical implication is that both MHI and VHI restore EELV to greater than baseline levels post-ES and that EELV remains above baseline thirty minutes post-hyperinflation. Therefore, our results suggest that either technique may be applied clinically post-ES to reverse suction-induced lung volume loss. Atelectrauma is one of the classic mechanisms of ventilator induced lung injury and is defined by Beitler et al [2] as "lung injury caused by high shear forces from 
cyclic opening and collapse of atelectatic but recruitable lung units". Our study suggests that ES induces atelectasis that is reversible. Hence, in the clinical setting where ES is performed regularly, it may be beneficial to recruit these alveoli to prevent atelectrauma.

While MHI generated a greater EELI during hyperinflation and at 5 minutes afterwards, it is interesting to observe that both groups were not different at 1 minute post hyperinflation. In the first minute post-VHI, EELI continued to improve whereas it declined post-MHI. We speculate that this observation reflects the loss of lung volume that occurs when the MV circuit is disconnected for any reason, in this case after performing MHI.

We observed that the second intervention, whether MHI or VHI, resulted in a smaller increase in EELI than the first. This is likely because the first manoeuvre successfully recruited alveoli, leaving fewer to recruit during the second manoeuvre. To minimise bias on the principal results of the study, the intervention order was randomised, new baseline data were established for each intervention and data analysis was performed in reference to baseline. However, due to the small sample size, it is not possible to exclude a casual over or under-estimation of the effect of one technique and a larger study may be needed.

While this study is the first to investigate the effects of MHI and VHI on restoring EELV post-ES, multiple previous studies $[10,11,15,20,21]$ have compared their effects on pulmonary compliance, oxygenation and haemodynamics. Consistent with these previous studies, we did not observe adverse haemodynamic events associated with MHI or VHI. When comparing pulmonary compliance (static, dynamic, or both) previous studies $[10,15,20,21]$ demonstrated no significant difference between MHI and VHI. Likewise, our study found no difference between groups. However, the trend of improvement in $\mathrm{C}_{\text {stat, }}$, from baseline shows that $\mathrm{MHI}$ was higher at 1 minute post-hyperinflation, 
whereas VHI was higher from 5 to 30 minutes. This trend is consistent with Savian et al [15] and Berney et al [21] who found that $\mathrm{C}_{\text {stat }}$ improved more at 30 minutes after VHI versus MHI. VHI may be superior to $\mathrm{MHI}$ in improving $\mathrm{C}_{\text {stat }}$ because there is no disconnection from the MV circuit, therefore, positive end-expiratory pressure is maintained. Nevertheless, our study demonstrated that both MHI and VHI at least restore $\mathrm{C}_{\text {stat }}$ to baseline, negating the deleterious effects of ES.

Previous studies have found no difference between the effects of $\mathrm{MHI}$ and $\mathrm{VHI}$ on $\mathrm{PaO}_{2} / \mathrm{FiO}_{2}[10,15$, 20]. Our results are consistent with these previous findings. When comparing to baseline, Dennis et al [10] found that $\mathrm{PaO}_{2} / \mathrm{FiO}_{2}$ ratio improved over time post-VHI whereas it decreased over time postMHI. Our study reflects the same trend in $\mathrm{PaO}_{2}$ and $\mathrm{PaO}_{2} / \mathrm{FiO}_{2}$ ratio at 30 minutes posthyperinflation, albeit with statistical non-significance. It may be hypothesised that a trend towards a more sustained improvement in oxygenation post-VHI is again due to the avoidance of MV circuit disconnection. Of key clinical importance though, is the outcome that gas exchange was not significantly impaired when MHI or VHI were implemented post-ES.

As discussed by Dennis et al [10] the effect of MHI may be overstated under study protocol conditions when compared to clinical practice. During the study, MHI was performed by an experienced clinician with a consistent technique including a 2 second inspiratory hold and PIP of 35$40 \mathrm{cmH}_{2} \mathrm{O}$ as measured by a manometer. In clinical practice, $\mathrm{MHI}$ is performed by clinicians of widely varying experience, commonly without a manometer. Previous studies have demonstrated that final year undergraduate physiotherapy students and intensive care nurses were unable to achieve target PIP or tidal volume while performing MHI $[32,33]$. In our intensive care unit, VHI is only performed clinically by experienced clinicians with a consistent procedure and accurate delivery of PIP to $35-40 \mathrm{cmH}_{2} \mathrm{O}$. Hence, the clinical effect of VHI is potentially more consistent with the study conditions. Furthermore, in clinical practice, VHI has potential advantages over MHI including greater accuracy of treatment parameters and avoidance of MV circuit disconnection. 
A large number of patients were excluded from the study. This was predominately due to contraindications of MHI, VHI or MV disconnection. Additionally, participants were excluded due to an inability to position them in 30 degrees of bed head elevation or contraindications to applying EIT. However, in clinical practice, the clinician can implement MHI or VHI exclusively according to their assessment, meaning that MV disconnection may not always be necessary. Furthermore, the clinician can choose any feasible patient position and is not restricted by the application of EIT. Therefore, we believe that our results are clinically applicable to a broader patient cohort.

There are limitations to this study which require discussion. The sample size is small, owing to a large number of exclusion criteria and pragmatic barriers to participant recruitment. However, we were able to demonstrate meaningful results in the primary outcome measure by using a randomised crossover design with a standardised washout period and establishing new baseline data prior to each intervention. The inclusion criteria contained specific restrictions regarding the duration of MV (4896 hours), the $\mathrm{PaO}_{2} / \mathrm{FiO}_{2}$ ratio (200-350mmHg) and the Riker score of sedation (2-3). These were designed to yield as homogenous a population as possible. However, they were later amended to facilitate participant recruitment rate. While this yielded a broader range of MV duration, baseline $\mathrm{PaO}_{2} / \mathrm{FiO}_{2}$ ratio remained mostly within the desired range (see Table 2). Three participants were recruited post Riker score amendment with one becoming slightly agitated during data collection, resulting in increased heart rate and mean arterial pressure values. By targeting participants who were sedated, we attempted to minimise the impact of variable spontaneous breathing. Inevitable spontaneous breaths did occur, however, by establishing new baseline data prior to each intervention and reporting on change from baseline, variability between patients was accounted for. Although $\mathrm{C}_{\text {stat }}$ was measured on the ventilator in real time, other values such as plateau pressure were not recorded which prevents retrospective analysis of driving pressure. It was not possible for the investigators 
delivering the intervention and recording data to be blinded. Steps were implemented in the participant randomisation process and delivery of the methodology to minimise biased outcome data.

Previous studies $[14,34,35]$ have recommended a recruitment manoeuvre during or post-ES to maintain oxygenation and prevent sustained lung collapse. Our results support the implementation of either MHI or VHI as a recruitment manoeuvre post-ES in this study population. However, the protocol described in our study may be arduous for clinical intensive care unit staff to apply routinely post-ES. Therefore, future research is warranted to determine the most efficient manoeuvre. For example, the majority of EELV may potentially be restored after just one minute of hyperinflation or five to six hyperinflation breaths. In clinical practice, MHI or VHI is often performed before and after ES, therefore it would be favourable to also demonstrate whether this is additionally protective of EELV post-ES. Furthermore, this study and previous studies $[6,7]$ have demonstrated significant lung de-recruitment post-ES of sedated or immobile patients. As mechanically ventilated patients are more rapidly woken and mobilised, it would be beneficial to investigate whether this effect is evident in patients who can breathe spontaneously, sigh, change position and cough.

\section{Conclusion}

Endotracheal suctioning of mechanically ventilated adults results in refractory loss of EELV. Our study demonstrates that both MHI and VHI are effective in restoring lung volume following ES and suggests that either technique should be applied clinically to minimise atelectrauma. Further research is warranted to determine the most time efficient MHI or VHI manoeuvre post-ES for translation into clinical practice. 
Declarations of interest: none

\section{Funding Support}

This work was supported by The Prince Charles Hospital Foundation (grant number NI2015-107). The funding source had no involvement in the study design; in the collection, analysis and interpretation of data; in the writing of the report; or in the decision to submit the article for publication.

\section{References}

1. Hodgson, C., et al., Recruitment manoeuvres for adults with acute respiratory distress syndrome receiving mechanical ventilation. Cochrane Database Syst Rev, 2016. 11: p. CD006667.

2. Beitler, J.R., A. Malhotra, and B.T. Thompson, Ventilator-induced Lung Injury. Clin Chest Med, 2016. 37(4): p. 633-646.

3. Albert, R.K., The role of ventilation-induced surfactant dysfunction and atelectasis in causing acute respiratory distress syndrome. Am J Respir Crit Care Med, 2012. 185(7): p. 702-8.

4. Lachmann, B., Open up the lung and keep the lung open. Intensive Care Med, 1992. 18(6): p. $319-21$.

5. $\quad$ Caironi, P., et al., Lung opening and closing during ventilation of acute respiratory distress syndrome. Am J Respir Crit Care Med, 2010. 181(6): p. 578-86.

6. Heinze, H., et al., Functional residual capacity changes after different endotracheal suctioning methods. Anesth Analg, 2008. 107(3): p. 941-4.

7. Corley, A., et al., End-expiratory lung volume recovers more slowly after closed endotracheal suctioning than after open suctioning: a randomized crossover study. J Crit Care, 2012. 27(6): p. 742 e1-7.

8. Liu, X.W., et al., Differential Effects of Endotracheal Suctioning on Gas Exchanges in Patients with Acute Respiratory Failure under Pressure-Controlled and Volume-Controlled Ventilation. Biomed Res Int, 2015. 2015: p. 941081.

9. Lasocki, S., et al., Open and closed-circuit endotracheal suctioning in acute lung injury: efficiency and effects on gas exchange. Anesthesiology, 2006. 104(1): p. 39-47.

10. Dennis, D., W. Jacob, and C. Budgeon, Ventilator versus manual hyperinflation in clearing sputum in ventilated intensive care unit patients. Anaesth Intensive Care, 2012. 40(1): p. 1429.

11. Anderson, A., et al., Effects of ventilator vs manual hyperinflation in adults receiving mechanical ventilation: a systematic review of randomised clinical trials. Physiotherapy, 2015. 101(2): p. 103-10.

12. Paulus, F., et al., Benefits and risks of manual hyperinflation in intubated and mechanically ventilated intensive care unit patients: a systematic review. Crit Care, 2012. 16(4): p. R145.

13. Clement, A.J. and S.K. Hubsch, Chest physiotherapy by the 'bag squeezing' method: a guide to technique. Physiotherapy, 1968. 54(10): p. 355-9.

14. Maggiore, S.M., et al., Prevention of endotracheal suctioning-induced alveolar derecruitment in acute lung injury. Am J Respir Crit Care Med, 2003. 167(9): p. 1215-24.

15. Savian, C., J. Paratz, and A. Davies, Comparison of the effectiveness of manual and ventilator hyperinflation at different levels of positive end-expiratory pressure in artificially ventilated and intubated intensive care patients. Heart Lung, 2006. 35(5): p. 334-41.

16. Hedenstierna, G., et al., Phrenic nerve stimulation during halothane anesthesia. Effects of atelectasis. Anesthesiology, 1994. 80(4): p. 751-60.

17. McCann, U.G., 2nd, et al., Visual validation of the mechanical stabilizing effects of positive end-expiratory pressure at the alveolar level. J Surg Res, 2001. 99(2): p. 335-42. 
18. Dennis, D.M., W.J. Jacob, and F.D. Samuel, A survey of the use of ventilator hyperinflation in Australian tertiary intensive care units. Crit Care Resusc, 2010. 12(4): p. 262-8.

19. Hayes, K., et al., Ventilator hyperinflation: a survey of current physiotherapy practice in Australia and New Zealand. New Zealand Journal of Physiotherapy, 2011. 39(3): p. 124-130.

20. Ahmed, F., et al., Comparison of effects of manual versus ventilator hyperinflation on respiratory compliance and arterial blood gases in patients undergoing mitral valve replacement. Heart Lung, 2010. 39(5): p. 437-43.

21. Berney, S. and L. Denehy, A comparison of the effects of manual and ventilator hyperinflation on static lung compliance and sputum production in intubated and ventilated intensive care patients. Physiother Res Int, 2002. 7(2): p. 100-8.

22. Kobylianskii, J., et al., Electrical impedance tomography in adult patients undergoing mechanical ventilation: A systematic review. J Crit Care, 2016. 35: p. 33-50.

23. Ukere, A., et al., Perioperative assessment of regional ventilation during changing body positions and ventilation conditions by electrical impedance tomography. $\mathrm{Br} \mathrm{J}$ Anaesth, 2016. 117(2): p. 228-35.

24. Frerichs, I., et al., Detection of local lung air content by electrical impedance tomography compared with electron beam CT. J Appl Physiol (1985), 2002. 93(2): p. 660-6.

25. Hinz, J., et al., End-expiratory lung impedance change enables bedside monitoring of endexpiratory lung volume change. Intensive Care Med, 2003. 29(1): p. 37-43.

26. van Genderingen, H.R., A.J. van Vught, and J.R. Jansen, Estimation of regional lung volume changes by electrical impedance pressures tomography during a pressure-volume maneuver. Intensive Care Med, 2003. 29(2): p. 233-40.

27. Grant, C.A., et al., The assessment of regional lung mechanics with electrical impedance tomography: a pilot study during recruitment manoeuvres. Intensive Care Med, 2009. 35(1): p. 166-70.

28. Tingay, D.G., et al., The effect of endotracheal suction on regional tidal ventilation and endexpiratory lung volume. Intensive Care Med, 2010. 36(5): p. 888-96.

29. Lindgren, S., et al., Regional lung derecruitment after endotracheal suction during volume- or pressure-controlled ventilation: a study using electric impedance tomography. Intensive Care Med, 2007. 33(1): p. 172-80.

30. Perneger, T.V., What's wrong with Bonferroni adjustments. BMJ, 1998. 316(7139): p. 12368.

31. Fernandez, M.D., et al., Changes in lung volume with three systems of endotracheal suctioning with and without pre-oxygenation in patients with mild-to-moderate lung failure. Intensive Care Med, 2004. 30(12): p. 2210-5.

32. Redfern, J., E. Ellis, and W. Holmes, The use of a pressure manometer enhances student physiotherapists' performance during manual hyperinflation. Aust J Physiother, 2001. 47(2): p. 121-31.

33. Glass, C., et al., Nurses' ability to achieve hyperinflation and hyperoxygenation with a manual resuscitation bag during endotracheal suctioning. Heart Lung, 1993. 22(2): p. 15865.

34. Dyhr, T., J. Bonde, and A. Larsson, Lung recruitment manoeuvres are effective in regaining lung volume and oxygenation after open endotracheal suctioning in acute respiratory distress syndrome. Crit Care, 2003. 7(1): p. 55-62.

35. Kasim, I., et al., A recruitment breath manoeuvre directly after endotracheal suction improves lung function: an experimental study in pigs. Ups J Med Sci, 2009. 114(3): p. 129-35. 
Figure legend

Figure 1: CONSORT diagram of patient flow through the study

Figure 2: Changes in mean EELI during ES, hyperinflation and at each time point post MHI and VHI

EELI = End-Expiratory Lung Impedance; ES = Endotracheal Suction; MHI = Manual Hyperinflation; VHI $=$ Ventilator Hyperinflation 
Table 1: Inclusion, Exclusion and Withdrawal Criteria

\section{Original Inclusion Criteria}

- $\quad \geq 18$ years

- Intra-arterial line in situ

- Mechanically ventilated on synchronised intermittent mandatory ventilation - volume control mode for 48 to 96 hours

- Ratio of arterial oxygen partial pressure to fraction of inspired oxygen $\left(\mathrm{PaO}_{2} / \mathrm{FiO}_{2}\right) 200$ to 350

- Sedated to Riker score of 2 to 3

\section{Amended Inclusion Criteria}

- $\quad \geq 18$ years

- Intra-arterial line in situ

- Mechanically ventilated on synchronised intermittent mandatory ventilation - volume control mode

\section{Exclusion Criteria}

- Agitated patients with a Riker score of 5-7

- Positive end expiratory pressure $>10 \mathrm{~cm} \mathrm{H}_{2} \mathrm{O}$

- Fraction of inspired oxygen $>60 \%$

- Peripheral oxygen saturation $<90 \%$

- Acute respiratory distress syndrome and/or lung protective ventilation

- Haemodynamically unstable (mean arterial pressure $<60 \mathrm{mmHg}$; resting heart rate $<60 \mathrm{~B} / \mathrm{min}$ or $>130 \mathrm{~B} / \mathrm{min}$; arrhythmias compromising blood pressure)

- Peak inspiratory pressure $>40 \mathrm{~cm} \mathrm{H}_{2} \mathrm{O}$ (as recorded on ventilator)

- Pneumothorax or air leak from chest drains

- Severe bronchospasm or gas trapping

- Frank haemoptysis

- Acute pulmonary oedema

- Acute head injury or suspected raised intra-cranial pressure

- Patients immediately post lung transplant or pulmonary thrombo-endarterectomy

- Patients unable to tolerate head of bed elevation of 30 degrees

- Open sternum

\section{Withdrawal Criteria}

- Haemodynamic instability during study intervention

- Sustained peak inspiratory pressure $>40 \mathrm{~cm} \mathrm{H}_{2} \mathrm{O}$ during study intervention

- Peripheral oxygen saturation $<90 \%$ during study intervention 
Table 2: Participant Demographics $(\mathrm{n}=9)$

\begin{tabular}{lc}
\hline Sex (male) & $8(88.8 \%)$ \\
Age (years) & $59.0(47.5$ to 67.0$)$ \\
BMI $\left(\mathbf{k g} / \mathbf{m}^{2}\right)$ & $28.0(26.0$ to 30.5$)$ \\
APACHE II Score at ICU admission & $22.0(12.0$ to 23.0$)$ \\
SOFA Score at time of study & $7.0(6.0$ to 9.5$)$ \\
Duration of mechanical ventilation at time of study (hours) & $70.0(28.5$ to 186.0$)$ \\
Baseline PaO $/$ FiO ${ }_{2}$ ratio (mmHg) & $242.1(210.5$ to 273.7$)$ \\
Primary Diagnosis: & \\
• Cardiac arrest/myocardial infarct & $3(33.3 \%)$ \\
- Sepsis & $2(22.2 \%)$ \\
- Neurological or metabolic disorder & $2(22.2 \%)$ \\
• Pneumonia & $1(11.1 \%)$ \\
- Cardiothoracic surgery & $1(11.1 \%)$ \\
\hline
\end{tabular}

Categorical data presented as $\mathrm{n}(\%)$. Continuous data presented as median (interquartile range). BMI $=$ Body Mass Index, APACHE II = Acute Physiology and Chronic Health Evaluation II, SOFA = Sequential Organ Failure Assessment, $\mathrm{PaO}_{2}=$ partial pressure of oxygen in arterial blood, $\mathrm{FiO}_{2}=$ fraction of inspired oxygen. 
Table 3: Baseline Ventilator Settings $(n=9)$

Ventilator mode

Fraction of inspired oxygen (\%)

Respiratory rate (breaths/minute)

Tidal volume (millilitres)

Positive end-expiratory pressure $\left(\mathrm{cmH}_{2} \mathrm{O}\right)$

Pressure support $\left(\mathrm{cmH}_{2} \mathrm{O}\right)$

Inspiratory flow rate (litres/minute)
Synchronised intermittent mandatory ventilation - volume control (SIMV-VC)

$$
40.5 \pm 5.5
$$

$13 \pm 5$

$554 \pm 65$

$7.3 \pm 1.7$

$10.2 \pm 0.6$

$52.5 \pm 5.4$

Data expressed as mean \pm standard deviation; $\mathrm{cmH}_{2} \mathrm{O}=$ centimetres of water 
Table 4: Changes in EELI (z) from baseline post MHI and VHI, and the difference between treatments

\begin{tabular}{cccc}
\hline Time Point & MHI & VHI & $\begin{array}{c}\text { Difference (MHI - } \\
\text { VHI) }\end{array}$ \\
\hline During hyperinflation & $1154(977$ to 1330$)$ & $769(457$ to 1080$)$ & $385(250$ to 520$)$ \\
1 minute & $852(614$ to 1089$)$ & $839(326$ to 1352$)$ & $307(27$ to 586$)$ \\
5 minutes & $835(589$ to 1080$)$ & $528(4$ to 1053$)$ & $174(-109$ to 456$)$ \\
15 minutes & $351(111$ to 592$)$ & $178(-346$ to 701$)$ & $114(-148$ to 377$)$ \\
\hline 0 minutes & $186(-38$ to 410$)$ & $72(-415$ to 558$)$ & \\
\hline
\end{tabular}

Data expressed as mean (95\% confidence interval); MHI = Manual Hyperinflation; VHI = Ventilator Hyperinflation; EELI = End Expiratory Lung Impedance 
Table 5: Changes in oxygenation variables from baseline post MHI and VHI

\begin{tabular}{|c|c|c|c|c|c|c|}
\hline & MHI & & & VHI & & \\
\hline Time point & $\mathrm{SpO}_{2}(\%)$ & $\mathrm{PaO}_{2}(\mathrm{mmHg})$ & $\begin{array}{c}\mathrm{PaO}_{2} / \mathrm{FiO}_{2} \\
(\mathrm{mmHg})\end{array}$ & $\mathrm{SpO}_{2}(\%)$ & $\mathrm{PaO}_{2}(\mathrm{mmHg})$ & $\mathrm{PaO}_{2} / \mathrm{FiO}_{2}(\mathrm{mmHg})$ \\
\hline 1 minute & $1.4(0.5$ to 2.3$)$ & 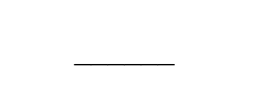 & 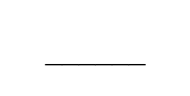 & $\begin{array}{c}1.3(-0.7 \text { to } \\
3.2)\end{array}$ & & \\
\hline 5 minutes & $\begin{array}{c}0.5(-0.4 \text { to } \\
1.4)\end{array}$ & 19.7 (4.6 to 34.9 ) & $\begin{array}{c}32.9(-3.3 \text { to } \\
69.1)\end{array}$ & $\begin{array}{c}0.3(-1.7 \text { to } \\
2.2)\end{array}$ & $\begin{array}{c}14.2(-18.4 \text { to } \\
46.85)\end{array}$ & $30.5(-47.5$ to 108.5$)$ \\
\hline 15 minutes & $\begin{array}{c}0.1(-0.8 \text { to } \\
1.1)\end{array}$ & & 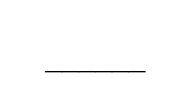 & $\begin{array}{c}-0.3(-2.2 \text { to } \\
1.7)\end{array}$ & & \\
\hline 30 minutes & $\begin{array}{c}0.1(-0.8 \text { to } \\
1.1)\end{array}$ & $-0.8(-16$ to 14.3$)$ & $\begin{array}{c}-28(-66.2 \text { to } \\
10.2)\end{array}$ & $\begin{array}{c}-0.3(-2.3 \text { to } \\
1.8)\end{array}$ & $\begin{array}{c}4.6(-28.04 \text { to } \\
37.22)\end{array}$ & $13.5(-68.2$ to 95.25$)$ \\
\hline
\end{tabular}

Data expressed as mean (95\% confidence interval); $\mathrm{MHI}=$ Manual Hyperinflation; $\mathrm{VHI}=$ Ventilator Hyperinflation; $\mathrm{SpO}_{2}=$ peripheral oxygen saturation; $\mathrm{PaO}_{2}=$ partial pressure of oxygen in arterial blood; $\mathrm{mmHg}=$ millimetres of mercury; $\mathrm{FiO}_{2}=$ fraction of inspired oxygen 


\section{Highlights}

- Endotracheal suction is associated with significant end-expiratory lung volume loss

- Manual and ventilator hyperinflation restore lung volume after endotracheal suction

- Both techniques have similar effects on oxygenation and lung compliance

- Ventilator hyperinflation is a safe alternative to manual hyperinflation 


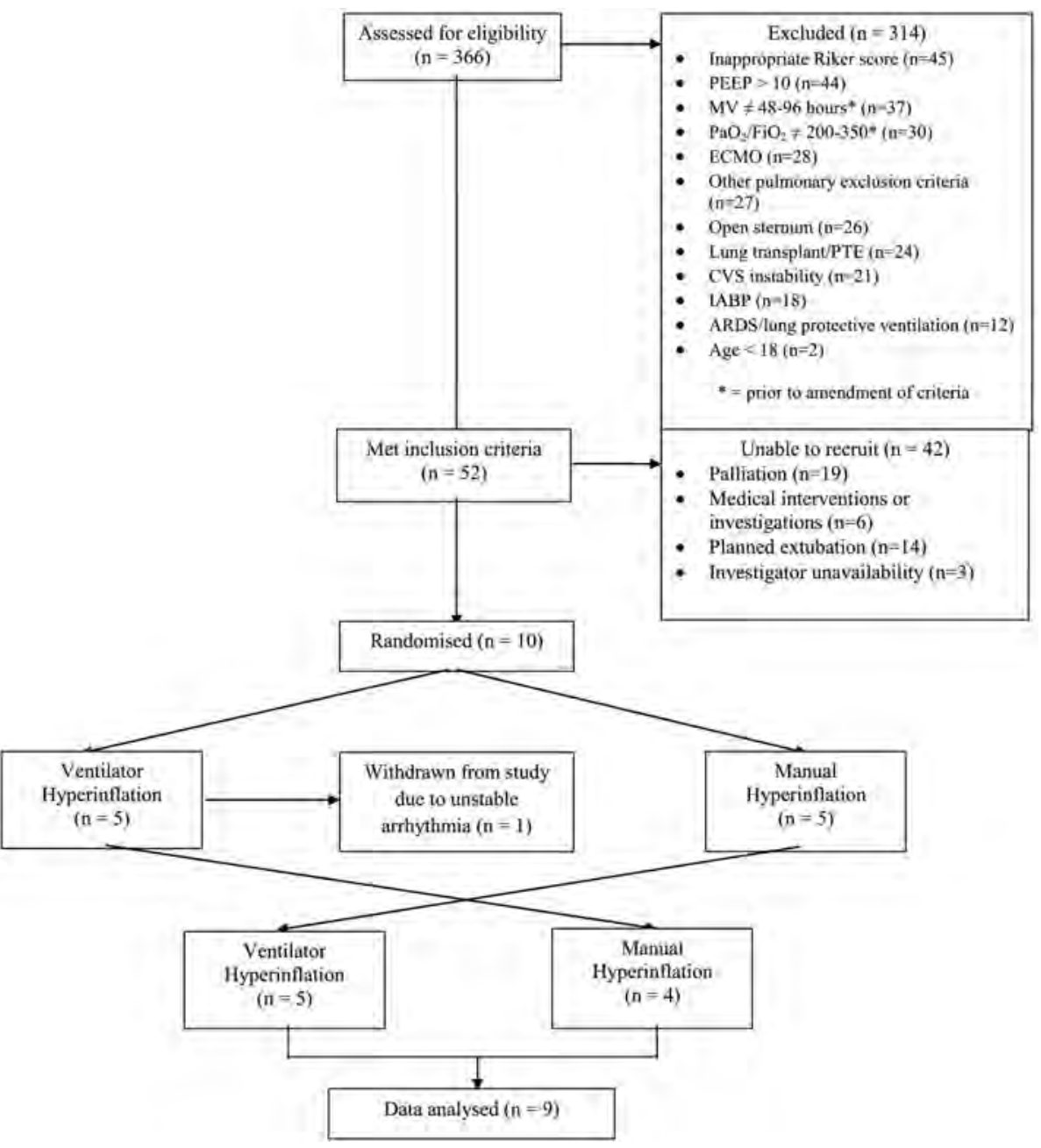

Figure 1 


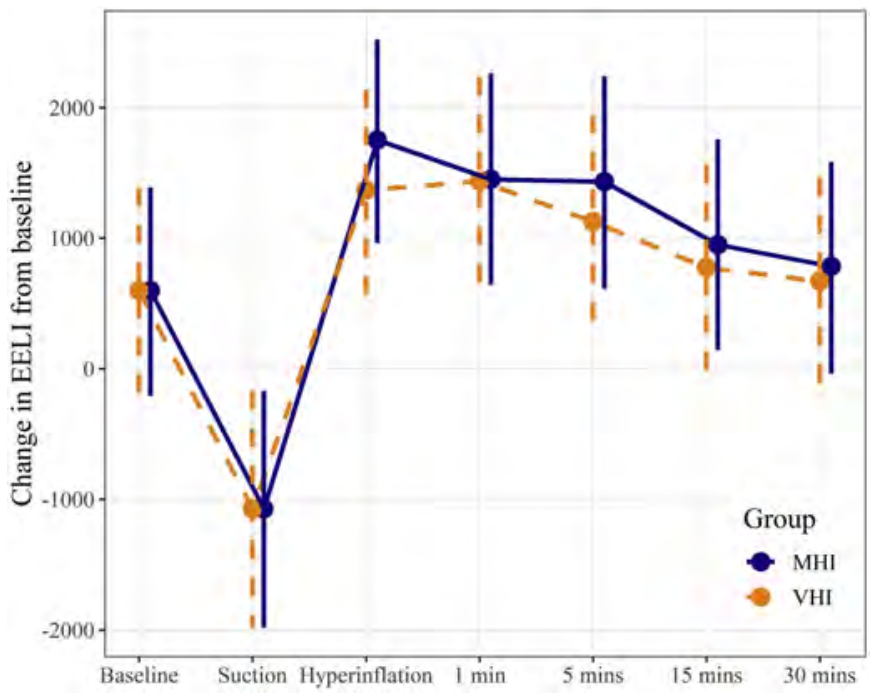

Figure 2 